\title{
Heat capacity of water: A signature of nuclear quantum effects
}

\author{
C. Vega ${ }^{1, a)}$ M. M. Conde,${ }^{1}$ C. McBride,${ }^{1}$ J. L. F. Abascal, ${ }^{1}$ E. G. Noya,${ }^{2}$ R. Ramirez,${ }^{3}$ and \\ L. M. Sesé \\ ${ }^{1}$ Departamento de Química Física, Facultad de Ciencias Químicas, Universidad Complutense de Madrid, \\ 28040 Madrid, Spain \\ ${ }^{2}$ Instituto de Química Física Rocasolano, Consejo Superior de Investigaciones Científicas (CSIC), \\ Calle Serrano 119, 28006 Madrid, Spain \\ ${ }^{3}$ Instituto de Ciencia de Materiales, CSIC, Campus de Cantoblanco, 28049 Madrid, Spain \\ ${ }^{4}$ Departamento de Ciencias y Técnicas Fisicoquímicas, Facultad de Ciencias, UNED, \\ Paseo Senda del Rey 9, 28040 Madrid, Spain
}

(Received 12 November 2009; accepted 3 January 2010; published online 22 January 2010)

[doi:10.1063/1.3298879]

Given the primordial role that water plays in life and our everyday lives, the development of high quality interaction potentials for this ubiquitous material is of great interest. Such models can be obtained "on the fly," as in CarrParrinello simulations, ${ }^{1}$ or by fitting the results of high level $a b$ initio calculations for clusters to an analytical expression. ${ }^{2}$ Another route is to use an empirical potential whose parameters are fine tuned so as to reproduce experimental properties. ${ }^{3}$ Once the potential energy surface (PES) is known computer simulations can be performed using either classical or quantum statistical mechanics to describe the probability density distribution of the molecules on this PES. Evidence that nuclear quantum effects in water are important is steadily growing. ${ }^{4-8}$ If the PES is obtained from first principles calculations then, since one is obtaining the true PES (although in an approximate manner), it is clear that quantum statistical mechanics should be used to describe the motion of the nuclei. In the case of empirical potentials one can use either classical or quantum statistical mechanics, depending on whether the potential parameters were obtained to reproduce experimental properties of water within classical or quantum simulations, respectively. Recently we have proposed two empirical potentials: the TIP4P/2005 (Ref. 9) to be used in classical simulations and TIP4PQ/2005 $5^{10,11}$ to be used in path-integral (PI) simulations. Both of these models are rigid and nonpolarizable.

In this note we shall focus on the heat capacity $C_{p}$ at constant pressure along the $p=1$ bar isobar for the two aforementioned models. For the TIP4P/2005 model the values of $C_{p}$ obtained from classical simulations for ice $\mathrm{I}_{h}$ and water have been reported previously. ${ }^{12,13}$ For TIP4PQ/2005 we shall calculate $C_{p}$ using the simulation results for the enthalpy taken from our recent work on the ice phases ${ }^{10}$ and water. ${ }^{11}$ These were obtained from PI simulations of water (ice) using 300 (432) molecules and isotropic (anisotropic) $N p T$ ensemble simulations. The Lennard-Jones part of the potential was truncated at $8.5 \AA$, adding long range corrections to both the energy and pressure, and using Ewald sums. The rigid-body rotational propagator was taken from the work of Müser and Berne. ${ }^{14}$ For the fluid phase we used $P=7$ replicas, and for ice $\mathrm{I}_{h}$ the number of replicas was selected such that $P T \approx 1500 \mathrm{~K}$. The heat capacity is obtained as the first derivative of the enthalpy with respect to the temperature at constant pressure. The enthalpies of the fluid phase (for seven temperatures) ${ }^{11}$ have been fitted variously to either a second or third order polynomial. For ice $\mathrm{I}_{h}$ the enthalpies (at $T=77,100,125,150,200,250$, and $273 \mathrm{~K}$ ) have been fitted to the expression $H=a+b T^{2}+c T^{3}$. This expression satisfies the third law of thermodynamics and provided an excellent description of the experimental enthalpies ${ }^{15}$ of ice $\mathrm{I}_{h}$ up to the melting temperature.

In Fig. 1 the experimental ${ }^{15}$ and TIP4PQ/2005 PI results for the heat capacity of ice $\mathrm{I}_{h}$ and water are presented. By including nuclear quantum effects it is, for the first time, possible to describe the $C_{p}$ of ice $\mathrm{I}_{h}$ and liquid water over a broad range of temperatures. On the other hand, results from classical simulations of TIP4P/2005 fail in describing $C_{p}$ for all temperatures considered in this work. Although the failure of the classical treatment at low $T$ was expected (such a treatment does not satisfy the third law so $C_{p}$ is not zero at
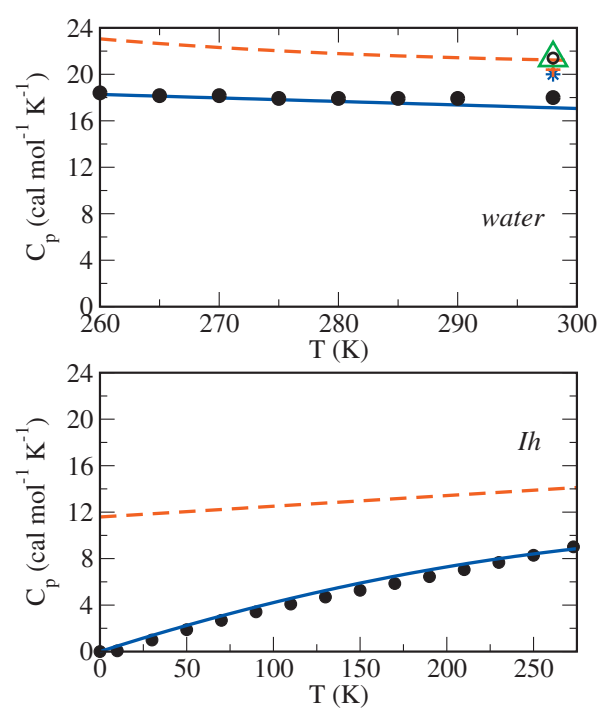

FIG. 1. Heat capacity $C_{p}$ at $p=1$ bar for water and ice $\mathrm{I}_{h}$ as obtained from PI simulations of TIP4PQ/2005 (solid line) and from classical simulations of TIP4P/2005 (dashed line). Experimental results (@); (water: Ref. 20, $\mathrm{I}_{h}$ : Ref. 15). Upper graph: fluid phase. Classical simulation results at room temperature for TIP4P-Ew $(\triangle)$, SPC/E $(\bigcirc)$, TIP4P $(+)$, and TIP3P $(*)$ are also shown. Lower graph: results for ice $\mathrm{I}_{h}$. 


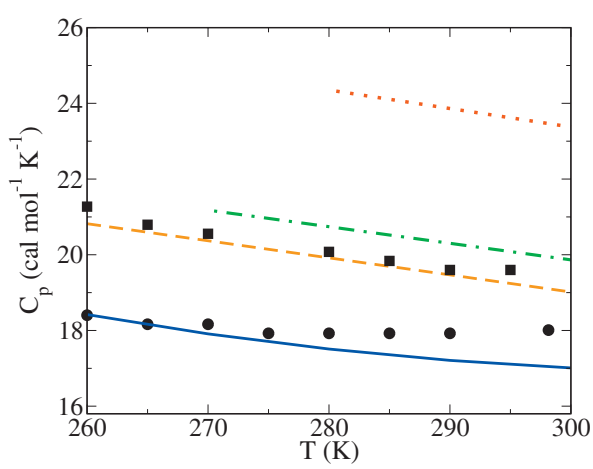

FIG. 2. $C_{p}$ from PI simulations of the TIP4PQ/2005 model for $\mathrm{H}_{2} \mathrm{O}$ (solid line), $\mathrm{D}_{2} \mathrm{O}$ (dashed line), and $\mathrm{T}_{2} \mathrm{O}$ (dashed-dotted line). The dotted line corresponds to the results obtained from classical simulations of TIP4PQ/ 2005. Experimental results of $C_{p}$ for $\mathrm{H}_{2} \mathrm{O}(\bullet)$ and $\mathrm{D}_{2} \mathrm{O}(\boldsymbol{\square})$ were taken from Ref. 20.

$0 \mathrm{~K}$ ) it was not obvious whether it should also fail at room temperature (for instance, with regard to density the third law only influences predictions below $125 \mathrm{~K}$; the TIP4P/ 2005 model results are rather good for densities from $150 \mathrm{~K}$ all the way up to the critical temperature). It is clear from the results presented in Fig. 1 that there is no temperature at which the classical description of $C_{p}$ provided by TIP4P/ 2005 is quantitatively correct (although predictions are certainly better at high temperatures). Also in Fig. 1 values of $C_{p}$ at room $T$ and $p$, obtained from classical simulations of several popular water models, are presented. ${ }^{16}$ Models reproducing the vaporization enthalpy of water [TIP3P, TIP4P (Ref. 17)] yield values of about $20 \mathrm{cal} / \mathrm{mol}$. Models that reproduce the vaporization enthalpy only when including the polarization term proposed by Berendsen et al. ${ }^{18}$ [TIP4P/Ew, ${ }^{19}$ TIP4P/2005, ${ }^{9}$ and SPC/E (Ref. 18)] yield a value of $C_{p}$ of about $21.5 \mathrm{cal} / \mathrm{mol}$. The TIP5P model yields $C_{p}=29 \mathrm{cal} / \mathrm{mol},{ }^{16}$ indicating an incorrect dependence of $H$ and $\rho$ with respect to $T$ (i.e., poor predictions for $C_{p}$ and for the thermal expansion coefficient) when the negative charge is situated on the "lone pair" electrons. From this it is clear that no model designed for classical simulations thus far is able to reproduce the value of $C_{p}$ at room $T$ and $p$, and that the inclusion of nuclear quantum effects clearly improves the predictions of $C_{p}$ for water.

To analyze whether the TIP4PQ/2005 model is also able to capture isotopic variations in $C_{p}\left(C_{p}\right.$ increases as the mass of the hydrogen isotope increases), in Fig. 2 we present the heat capacity for liquid $\mathrm{H}_{2} \mathrm{O}, \mathrm{D}_{2} \mathrm{O}$, and $\mathrm{T}_{2} \mathrm{O}$, along with experimental results for $\mathrm{H}_{2} \mathrm{O}$ and $\mathrm{D}_{2} \mathrm{O} .{ }^{20}$ One can see that the TIP4PQ/2005 model is also able to reproduce $C_{p}$ for $\mathrm{D}_{2} \mathrm{O}$. From a comparison of classical and quantum simulations of TIP4PQ/2005 water, it can be seen that nuclear quantum effects modify the value of $C_{p}$ by up to $6.5 \mathrm{cal} / \mathrm{mol}$. Classical simulations of TIP4P/2005 (at room $T$ and $p$ ) provide $C_{p}=21.1 \mathrm{cal} / \mathrm{mol}$, which differs from experiment by $\approx 3.1 \mathrm{cal} / \mathrm{mol}$ ( rather than $6.5 \mathrm{cal} / \mathrm{mol}$, which is the difference between quantum and classical results of TIP4PQ/2005). This indicates that the classical TIP4P/2005 model implicitly incorporates some nuclear quantum effects within the values of its parameters.
From the point of view of further improvements it is likely that intramolecular degrees of freedom (i.e., flexibility) should be included in the model, since these provide a small, but probably significant, contribution to intermolecular interactions. However, for a flexible model of water large differences in $C_{p}$ between the classical and the quantum treatment are still expected; ${ }^{21}$ internal vibrational modes would contribute little to $C_{p}$ in a quantum treatment, whereas they would contribute $3 R$ in a classical treatment. The value of $12.5 \mathrm{cal} / \mathrm{mol}$ (i.e., $6.5+3 R$ ) is probably an upper bound for the difference in $C_{p}$ between a classical and a quantum treatment ${ }^{21}$ of a flexible model of water. This is due to the existence of competing quantum effects (i.e., a lower dipole moment of water in the classical treatment) as discussed recently by Habershon et al., ${ }^{7}$ which would most likely narrow the gap between quantum and classical results.

In this note we have shown that by including nuclear quantum effects it is possible to provide a good description of $C_{p}$ of water and ice $\mathrm{I}_{h}$. The failure of all of the classical models to describe the heat capacity of water clearly shows that only a quantum treatment can quantitatively reproduce this property. The heat capacity is indeed one of the signatures of nuclear quantum effects in water.

This work has been funded by MEC (Grant Nos. FIS2007-66079-C02-01 and FIS2006-12117-C03), by CAM (Grant No. P2009/ESP-1691), and by UCM (Grant No. 910570).

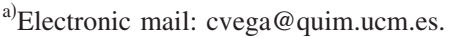

${ }^{1}$ R. Car and M. Parrinello, Phys. Rev. Lett. 55, 2471 (1985).

${ }^{2}$ G. S. Fanourgakis and S. S. Xantheas, J. Chem. Phys. 128, 074506 (2008).

${ }^{3}$ C. Vega, J. L. F. Abascal, M. M. Conde, and J. L. Aragones, Faraday Discuss. 141, 251 (2009).

${ }^{4}$ R. A. Kuharski and P. J. Rossky, Chem. Phys. Lett. 103, 357 (1984).

${ }^{5}$ L. Hernández de la Peña, M. S. G. Razul, and P. G. Kusalik, J. Chem. Phys. 123, 144506 (2005).

${ }^{6}$ F. Paesani and G. A. Voth, J. Phys. Chem. B 113, 5702 (2009).

${ }^{7}$ S. Habershon, T. E. Markland, and D. E. Manolopoulos, J. Chem. Phys. 131, 024501 (2009).

${ }^{8}$ J. A. Morrone and R. Car, Phys. Rev. Lett. 101, 017801 (2008).

${ }^{9}$ J. L. F. Abascal and C. Vega, J. Chem. Phys. 123, 234505 (2005).

${ }^{10}$ C. McBride, C. Vega, E. G. Noya, R. Ramírez, and L. M. Sesé, J. Chem. Phys. 131, 024506 (2009).

${ }^{11}$ E. G. Noya, C. Vega, L. M. Sesé, and R. Ramírez, J. Chem. Phys. 131, 124518 (2009).

${ }^{12}$ E. G. Noya, C. Menduiña, J. L. Aragones, and C. Vega, J. Phys. Chem. C 111, 15877 (2007).

${ }^{13}$ H. L. Pi, J. L. Aragones, C. Vega, E. G. Noya, J. L. F. Abascal, M. A. Gonzalez, and C. McBride, Mol. Phys. 107, 365 (2009).

${ }^{14}$ M. H. Müser and B. J. Berne, Phys. Rev. Lett. 77, 2638 (1996).

${ }^{15}$ R. Feistel and W. Wagner, J. Phys. Chem. Ref. Data 35, 1021 (2006).

${ }^{16}$ W. L. Jorgensen and J. Tirado-Rives, Proc. Natl. Acad. Sci. U.S.A. 102, 6665 (2005).

${ }^{17}$ W. L. Jorgensen, J. Chandrasekhar, J. D. Madura, R. W. Impey, and M. L. Klein, J. Chem. Phys. 79, 926 (1983).

${ }^{18}$ H. J. C. Berendsen, J. R. Grigera, and T. P. Straatsma, J. Phys. Chem. 91, 6269 (1987).

${ }^{19}$ H. W. Horn, W. C. Swope, J. W. Pitera, J. D. Madura, T. J. Dick, G. L. Hura, and T. Head-Gordon, J. Chem. Phys. 120, 9665 (2004).

${ }^{20}$ C. A. Angell, M. Oguni, and W. J. Sichina, J. Phys. Chem. 86, 998 (1982).

${ }^{21}$ M. Shiga and W. Shinoda, J. Chem. Phys. 123, 134502 (2005).
} 